\title{
Reconstruction and analysis of hybrid composite shells using meshless methods
}

\author{
G. M. S. Bernardo ${ }^{1}$ M. A. R. Loja ${ }^{1,2}$
}

Received: 25 May 2016/ Accepted: 30 January 2017/Published online: 16 February 2017

(c) The Author(s) 2017. This article is published with open access at Springerlink.com

\begin{abstract}
The importance of focusing on the research of viable models to predict the behaviour of structures which may possess in some cases complex geometries is an issue that is growing in different scientific areas, ranging from the civil and mechanical engineering to the architecture or biomedical devices fields. In these cases, the research effort to find an efficient approach to fit laser scanning point clouds, to the desired surface, has been increasing, leading to the possibility of modelling as-built/as-is structures and components' features. However, combining the task of surface reconstruction and the implementation of a structural analysis model is not a trivial task. Although there are works focusing those different phases in separate, there is still an effective need to find approaches able to interconnect them in an efficient way. Therefore, achieving a representative geometric model able to be subsequently submitted to a structural analysis in a similar based platform is a fundamental step to establish an effective expeditious processing workflow. With the present work, one presents an integrated methodology based on the use of meshless approaches, to reconstruct shells described by points' clouds, and to subsequently predict their static behaviour. These methods are highly appropriate on
\end{abstract}

M. A. R. Loja

amelialoja@dem.isel.ipl.pt

G. M. S. Bernardo

goncalo.bernardo@tecnico.ulisboa.pt

1 GI-MOSM, Grupo de Investigação em Modelação e Optimização de Sistemas Multifuncionais, ISEL, IPL, Instituto Superior de Engenharia de Lisboa, Av. Conselheiro Emídio Navarro 1, 1959-007 Lisbon, Portugal

2 LAETA, IDMEC, Instituto Superior Técnico, Universidade de Lisboa, Av. Rovisco Pais 1, 1049-001 Lisbon, Portugal dealing with unstructured points clouds, as they do not need to have any specific spatial or geometric requirement when implemented, depending only on the distance between the points. Details on the formulation, and a set of illustrative examples focusing the reconstruction of cylindrical and double-curvature shells, and its further analysis, are presented.

Keywords Points clouds - Meshless methods - Structural behaviour - Fibre reinforced composite materials . Nanocomposites

\section{Introduction}

During recent years, the use of 3D data acquisition devices has greatly increased resulting in the widespread dissemination of point clouds representations of sampled realworld objects. This reality denotes the importance of a greater investment in the research of efficient and robust approaches that provide not only the possibility of reconstruct surfaces from these point clouds, but also the possibility of further analysis of the surface without changing substantially the basis of the approach used in the first problem.

In this context, recent works (Huber et al. 2010; Bosch et al. 2014) have mentioned the potential of 3D laser scanning and Building Information Models (BIM) in fields, such as civil engineering, architecture, and construction, where frequent access to detailed and semantically rich information of as-built and as-is status of construction objects is required. Therefore, the use of acquisition data devices, such as laser scanners, can provide remarkable benefits if efficient approaches to handle, process, and store the information obtained are developed and implemented. 
It is also worth of mention that, normally, these 3D acquisition processes typically produce point clouds which are incomplete, noisy, and non-uniformly sampled. These characteristics grant to the modelling processes and methods, requirements associated with pre-processing techniques used to eliminate as much as possible the sampling errors, varying sampling density, and registration errors, i.e., find feasible modelling processes departing from unorganized 3D laser scanning point clouds. To note that concerning to the actual state-of-the-art, one is far from getting an immediate digital representation of the physical surface/component using an entirely automatic procedure.

From the literature review carried out, it is possible to notice that there are several possible techniques to obtain surfaces from ordered point clouds which, typically, find a single-curve segment that approximates or interpolates the given points, fitting the curve to the points by minimizing an error criterion (Rusinkiewicz and Levoy 2000). If points are ordered, piecewise polynomial curves can also be fitted to them. Difficulties arise, however, when the points have random/unknown positions. In recent years, some researchers have proposed approaches to obtain surfaces from unorganized points. Another work focused on the reconstruction of point set surfaces from point clouds based on the method of moving least squares was proposed by Alexa et al. (2001). Further approaches following the idea of locally polynomial surface patches to confined point neighbourhoods are proposed in Nealen (2004). Hoppe et al. (1992) described an algorithm for the reconstruction of a polyhedral surface from an unorganized set of points, which is based on region growing. They used a plane that is fitted to a neighbourhood around each data point, providing an estimate of the surface normal for the point. The surface normals are propagated using a minimal spanning tree, and then, a signed-distance function is constructed in small vicinities around the data points. In his works, Taubin (1991, 1993) reconstructed the surfaces by global algebraic fitting. The author fitted a polynomial implicit function to a point set by minimizing the distance between the point set and the implicit surface, and developed a first-order approximation of a Euclidean distance to improve later the approximation.

Alternative approaches based on implicit representation of object surfaces with radial basis functions (RBF) were presented by Carr et al. $(1997,2001)$ and Beatson et al. (2001). In these works, the authors used these functions to reconstruct cranial bone surfaces from 3D CT scans. Data surrounding large irregular holes in the skull were interpolated using thin-plate spline RBF. In Carr et al. (1997, 2001), the authors mentioned that these functions offer several advantages over piecewise polynomial interpolants and may simplify problems related to the process of smoothing and re-meshing existing noisy surfaces. New methods of surface reconstruction were presented by Turk and Obrien (2002), Dinh et al. (2002), and Morse et al. (2001), being specially relevant to highlight the one developed by Morse and his colleagues in which they proposed an algebraic method for creating implicit surfaces using linear combinations of radial basis interpolants to form complex models from scattered surface points. In their work, they explored and extended implicit interpolations methods to develop approaches computationally less complex, and more suitable for systems of large numbers of scattered surface points using compactly supported radial basis interpolants.

The use of different reconstruction techniques and its potential use with finite-element commercial applications have drawn the attention of a few researchers more recently. Among the works published on this subject, we may note the work carried out by Barazzetti et al. (2015), which described the use of BIM models derived from point clouds for structural simulation based on finite-element analysis. BIM interoperability has reached a significant level of development and detail, which is enabled by the great number and density of laser point clouds. However, from the finite-element analysis, this may be an effective drawback, thus requiring the rationalization of the BIM, without a drastic reduction of the BIM model, using tetrahedral meshes for finite-element analysis. In another work carried out by Yang et al. (2014), the authors studied the health assessment of concrete structures with a finiteelement model created in Ansys, based on the information obtained by terrestrial laser scanning (TLS). The authors concluded that the benefit of TLS lied mainly in the possibility of a surface-based validation of results predicted by the finite-element analyses. A semi-automatic procedure to transform three-dimensional point clouds of complex objects to three-dimensional finite-element models was presented by Castellazzi et al. (2015). When the starting point clouds represent the inner and outer surfaces of the scanned structure, the finite-element model obtained will represent the solid 3D structure. In a related work, Szolomicki (2015) considered the application of 3D scanning to create a numerical model of historical buildings, and not just as used to build inventory documentation with the visualization of a building and the corresponding creation of 3D models. To this purpose, the authors used computeraided design (CAD) programs to create an appropriate numerical model for subsequent static finite-element analysis (FEA). In addition, in other fields of knowledge, these approaches are becoming more familiar. An illustration of this is the review work carried out by Wittek et al. (2016). In that work, they have envisaged advances in computing and engineering technologies that may extend surgeons' ability to plan and carry out surgical interventions. They focused on methods for the generation of 
patient-specific computational grids used for solving partial differential equations governing the mechanics of the body organs. Another subject considered is related to the methods used for the identification and assignment of patientspecific material properties to tissues and boundary conditions. The authors carried a state-of-the-art in this area and enumerated asset of topics which may deserve the development of future research. In the context of the finiteelement analyses, namely concerning to the static and dynamic analysis of plates using meshfree methods; in the last years, one can refer the work, developed by Ferreira et al. (2005) in which multiquadric RBF were used. In that work, it was carried out the static analysis of a simply supported functionally graded plate modelled by a thirdorder shear deformation theory. More recently, Tornabene et al. (2013) presented a work, applying RBF to a general higher order equivalent single-layer formulation for the free vibrations of doubly curved laminated composite shells and panels. The theoretical development was based on Carrera Unified Formulation, in particular by deducting and defining explicitly the fundamental nuclei of a multilayered double-curved shell structure.

In the present paper, one proposes an integrated methodology based on meshless approaches and using radial basis functions. These functions are used to reconstruct the surfaces of the hybrid composite shells and to perform their static analyses. However, due to specific requirements and characteristics (Bernardo and Loja 2015a, b) of the problems to solve and to distinct methods and their achievements, in the first phase of this work, one uses radial basis functions with compact-support (CS-RBF) and in the second phase, one uses multiquadric functions.

The results obtained allow concluding on the good performance of this meshless integrated methodology devoted to reconstruction and structural analysis.

\section{Radial basis functions interpolation}

Concerning to the method used to solve the problem associated with the surface reconstruction, in this work, one constructs a signed-distance function to avoid the trivial solution (Carr et al. 2001; Bernardo et al. 2015), that function $\mathrm{f}$ which defines implicitly the surface is zero everywhere. The associated problem is: given $n$ distinct points $\left\{\left(x_{i}, y_{i}, z_{\mathrm{i}}\right)\right\}_{i=1}^{n}$ on a surface $M$ in $\Re^{3}$, find a surface $M^{\prime}$ that is a reasonable approximation to $M$ in $\Re^{3}$. If the surface $M$ consists of all the points $(x, y, z)$ that satisfy the equation:

$f(x, y, z)=0$,

then we say that $f$ implicitly defines $M$. Here, we want to approximate a signed-distance function given a set of zero- valued surface points and non-zero off-surface, leading to a problem that can be stated as follows: given a set of distinct nodes, $X=\left\{x_{i}\right\}_{i=1}^{N} \subset \Re^{3}$, find an interpolant $s:\left\{f_{i}\right\}_{i=1}^{N} \subset R$ such that

$s\left(x_{i}\right)=f_{i} \quad i=1, \ldots, N$.

In addition, the smoothest interpolant $s(x)$ has the simple form (particular example of an RBF) given by:

$s(x)=p(x)+\sum_{i=1}^{N} \lambda_{i} \Phi\left(\left|x-x_{i}\right|\right)$,

where the second part of the equation can be one of the RBF functions mentioned in Carr et al. (1997, 2001), Bernardo and Loja 2015), $p$ has a low degree and the RBF is a real-valued function on $[0, \infty)$, usually unbounded and with a compact-support (Wendland 1995). In this context, the points $x_{i}$ are referred to as the centres of the RBF.

Radial basis functions are popular for interpolating scattered data, as the associated system of linear equations is guaranteed to be invertible under very smooth conditions on the locations of the data points (Cheney and Light 1999; Savchenko et al. 1995). In general, if the polynomial in Eq. (3) is of degree $m$, then it has to be implied that the side conditions (orthogonality) imposed on the coefficients $\lambda_{i}$ are

$\sum_{i=1}^{N} \lambda_{i} q\left(x_{i}\right)=0$,

for all polynomials $q$ with $m$ being its highest degree. This leads to a linear system, which is solved to obtain the coefficients that specify the RBF. Let $\left\{p_{1}, \ldots, p_{l}\right\}$ be a basis for polynomials with highest degree $m$, and let $c=\left\{c_{1}, \ldots, c_{l}\right\}$ be the coefficients. Then, Eqs. 2 and 4 may be written as follows:

$\left[\begin{array}{cc}A & P \\ P^{\mathrm{T}} & 0\end{array}\right]\left[\begin{array}{c}A \\ 0\end{array}\right]=B\left[\begin{array}{l}\lambda \\ c\end{array}\right]=\left[\begin{array}{l}f \\ 0\end{array}\right]$,

where

$A_{i, j}=\Phi\left(\left|x_{i}-x_{j}\right|\right), \quad i, j=1, \ldots, N$

$P=\left[\begin{array}{ccccc}1 & x_{1} & y_{1} & & p_{1}\left(x_{1}\right) \\ 1 & x_{2} & y_{2} & \cdots & p_{1}\left(x_{2}\right) \\ 1 & x_{3} & y_{3} & & p_{1}\left(x_{3}\right) \\ & \vdots & & \ddots & \vdots \\ 1 & x_{n} & y_{n} & \cdots & p_{1}\left(x_{n}\right)\end{array}\right]$.

By solving the linear system (5), one determines $\lambda$ and $c$, and hence $s(x)$. However, the matrix B in Eq. 5 is typically bad conditioned as the number of data points $N$ gets larger. This means that substantial errors will easily creep into any standard numerical solution.

However, due to the reasons mentioned in Bernardo and Loja (2015), in this work, we interpolate the compactly 
supported radial basis functions, which are briefly described in the following subsection. In addition, the terms $p_{i, j}$ are not considered.

\section{Shells surface reconstruction}

\section{Construction of a signed-distance function to fit a surface}

To fit an implicit function to a surface, one has to find a function $f$ which implicitly defines a surface $M^{\prime}$ and satisfies the following equation:

$f\left(x_{i}, y_{i}, z_{i}\right)=0 \quad i=1, \ldots, n$

where $\left\{\left(x_{i}, y_{i}, z_{i}\right)\right\}_{i=1}^{n}$ are points lying on the surface. To avoid the trivial solution, off-surface points are appended to the input data and are given non-zero values. This gives the interpolation problem:

find $f$ such that:

$f\left(x_{i}, y_{i}, z_{i}\right)=0, \quad i=1, \ldots, n, \quad$ (on-surface points)

$f\left(x_{i}, y_{i}, z_{i}\right)=d_{i} \neq 0, \quad i=n+1, \ldots, N, \quad$ (off-surface points).

This still leaves the problem of generating the off-surface points $\left\{\left(x_{i}, y_{i}, z_{i}\right)\right\}_{i=n+1}^{n}$ and the corresponding values $d_{i}$. A possible selection for $f$ is a signed-distance function, where the $d_{i}$ are chosen to be the distance to the closest onsurface point. Points outside the object are assigned positive values, while points inside the object are assigned negative values. These off-surface points which may be assigned either side of the surface (Fig. 1) are generated from projections along surface normals.

Due to the increase of points, one has to estimate the surface normals and to determine the appropriate projection distance. In the case of a mesh, it would be straightforward to define off-surface points, since normals are implied by the mesh connectivity at each vertex. However, in the present case, we have unorganized points-cloud data, and thus, normals will have to be estimated from a local neighbourhood of points.

\section{Principal component analysis for normal estimation}

In the present work, the normal at a point is estimated, by choosing a set of neighbour points close to the point considered. This proximity is determined upon a given search radius. The search is performed by an algorithm based on the construction of a $k d$ tree (Bentley 1975), containing the information of the nearest points of each point $p_{i}$, structured in a tree that keeps the positions of the points (right, left, top, or down). The unitary normal vector of the plane is determined via principal component analysis (PCA), (Hoppe et al. 1992; Chalmovianský and Juttler B 2003) by carrying the three main steps:

- Search for all points within a certain point neighbourhood $p_{i} \in P$,

- Estimation of the direction of the normal $n_{i}$ for $p_{i}$ via PCA,

- Consistent orientation of the generated normals $n_{i}$.

With respect to the first step, one can say that the neighbourhood of each point $p_{i} \in P$ consists of the $k$ nearest neighbours in the data set, that is

$P_{i}=\left\{p_{i, 0}, \ldots, p_{i, k-1}\right\}$.

Finally, one selects the scalar product $n_{i} \cdot n_{j}$ as the criterion to check if the normals are well oriented comparing to the nearest neighbourhood points, i.e., if the resulting value is far from the unit value, the normal of this point is not computed, and consequently, the normal points to the point are not computed either. However, one has to bear in mind that this method of seeking the normal vectors may lead to some significant errors if the chosen points to estimate the normals are not so close to the candidate points. In general, it is difficult to achieve a robust estimation of the normals everywhere. If normal direction or sense is ambiguous at a particular point, then a normal is not fitted at that point, instead that point is left as a "zeropoint" (just lied in the surface). Once the normals are computed, the position of the off-points created toward the direction of those normals can be calculated as follows:
Fig. 1 Illustration of the off and on-surface points

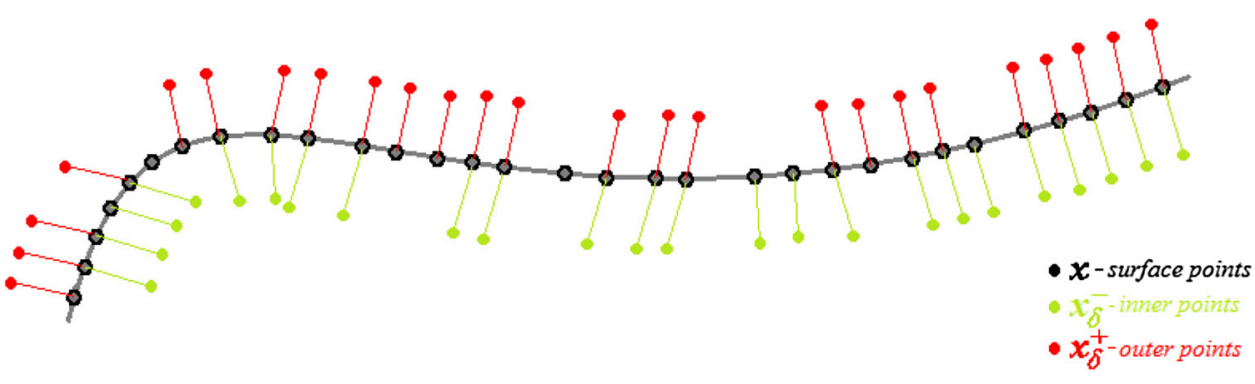




$$
\begin{aligned}
& \left(x_{N+i}, y_{N+i}, z_{N+i}\right)=p_{i}+\delta n o_{i} \\
& \quad=\left(x_{i}+\delta n o_{i}^{x}, y_{i}+\delta n o_{i}^{y}, z_{i}+\delta n o_{i}^{z}\right) \\
& \left(x_{2 N+i}, y_{2 N+i}, z_{2 N+i}\right)=p_{i}-\delta n o_{i} \\
& \quad=\left(x_{i}-\delta n o_{i}^{x}, y_{i}-\delta n o_{i}^{y}, z_{i}-\delta n o_{i}^{z}\right),
\end{aligned}
$$

where $\delta$ is the distance considered, between the in-surface points and the corresponding off-surface points, and $n o_{i}^{x, y, z}$, $i=1, \ldots, n$, denote the normals estimated for each coordinate axis.

This step consists on determining the value of the function $f$ whose zero contour (isosurface $f=0$ ) interpolates the point cloud data $x_{1}, \ldots, n$, and whose isosurface $f=1$ and $f=-1$ interpolate $x_{n+1, \ldots, 2 n}^{+}$and $x_{2 n+1, \ldots, 3 n}^{-}$, respectively, that is

$f\left(x_{i}\right)=\left\{\begin{array}{cc}0 & i=1 \ldots n \\ 1 & i=n+1 \ldots 2 n \\ -1 & i=2 n+1 \ldots 3 n .\end{array}\right.$

The values of \pm 1 for the auxiliary data are assigned in an arbitrary way. Such choice does not affect the quality of the results, as we are interested in the zero isosurface of $f$.

\section{Compactly supported radial basis functions}

As previously mentioned, in the present work, we use compactly supported (CS-RBF) as interpolant functions to construct the linear system of equations. We made this option, based on the literature review carried out, which pointed Wendland CS-RBF functions as being feasible choices as interpolant functions once they guarantee the creation of sparse and positive-definiteness matrices of the linear system (Wendland 1995). In general, the solutions of the minimum-degree polynomial for compact, locally supported radial basis functions have the form:

$f\left(x_{i}\right)=\left\{\begin{array}{cc}(1-r)^{p} P(r) & r<1 \\ 0 & \text { otherwise. }\end{array}\right.$

The computation of the function considers Shepard's Method, which is known to build interpolations and approximations with good properties under the present conditions. In fact, in these cases, the grid is composed by scattered nodes, and the basis reproduces exactly complete linear polynomials being the method applicable in any number of spatial dimensions. Shepard's Method is used here also due to its benefits that include better conditioning of discrete equations and easier handling of essential boundary conditions in applications to PDE. Furthermore, compared to moving least-squares approximations, the construction of the basis is quite fast (Krysl and Belytshko 2000). In this method, the influence domains $\Omega_{I}$ can be of any shape and a weight function $w_{i}(x)$ is associated with each node $i$. Its value is non-negative inside $\Omega_{I}$, vanishes on the boundary $\delta \Omega_{I}$, and is non-zero at the node. An approximation can be written as a linear combination, according to:

$u_{k}(x)=\sum_{i=1}^{N} \omega_{i}(x) u_{i}$

where $u_{i}$ are the nodal parameters, and $\omega_{i}(x)$ are the basis functions of compact-support, which are constructed from the weight functions associated with the nodes, $w_{i}(x)$

$\omega_{i}(x)=\frac{w_{i}(x)}{\sum_{k=1}^{N} w_{k}(x)}$.

Wendland (1995) has derived radial basis functions for various degrees of continuity $\left(C^{k}\right)$ and dimensionality (d) of the interpolated function. These functions have a unitary radius of support, but they can be scaled to allow any desired radius of support $\alpha$. The system of equations using the CS-RBF has the same form as Eq. 6, but the construction of the linear system considering that type of interpolant makes use of an important feature of RBF functions, namely the fact that they have a finite support.

\section{Multidimensional binary search tree}

A $k d$ tree is a multidimensional binary tree which grants good flexibility on performing different types of queries, and it has the following sorting property for a tree with point $\bar{x}$ at the root and subtrees $T_{\text {left }}$ and $T_{\text {right }}$ :

$\forall \bar{y} \in T_{\text {left }}: \bar{y}^{d} \leq x^{d}$

$\forall \bar{y} \in T_{\text {right }}: \bar{y}^{d} \geq x^{d}$,

where the sorting dimension $d$ changes at each level of the tree. $k d$ trees can be used to find all points within distance $\mathrm{r}$ of a particular constraint (Bentley 1975). While a number of points must still be tested explicitly, the multidimensional sorting nature of the $k d$ tree allows a large number of points to be rejected at each level of the tree (Morse et al. 2001). The resulting matrix is extremely sparse (Bernardo and Loja 2015). In the present work, we perform a query, that search for each surface point considered, the nearest neighbour points by considering the distance between the points (represented by a radius). The linear system of equations is then solved iteratively, considering in each loop a particular centre $\bar{c}_{i}$, with a corresponding neighbourhood.

\section{Selection of radius of support and isosurface extraction}

Due to the finite extent of CS-RBF, only those points within the radius of support of one of the original positions have non-zero values. Hence, it is crucial to properly select that radius of support to achieve optimal efficiency of 
computation and results. Too small a radius can produce basis functions that are unable to span the inter-constraint gaps. Too large a radius does not adversely affect the results but reduces the sparseness of the matrix, thus increasing the computation required. It is necessary to select a radius of support that is both large enough to produce effective results and not so large that the computation becomes impractical.

For all the points outside the radius of support, considered in each iteration of the surface reconstruction, all the terms in Eq. 4, with exception to the polynomial term, are zero. In this way, these embedding functions are not the same as those normally used for implicit surfaces-the implicit surface represented is not the only set of zerovalued points in the space. However, the implicit surface does form a unique contiguous locus of zero-valued points passing through the constraints. An isosurface extractor may be used to extract this surface by seeding it with any one of the initial constraints. To note that, care must be taken, so that the step size of the extractor does not cause it to jump outside the band of non-zero points. It is easy to explicitly recognize when no non-zero terms are found in Eq. 4 (as none of the constraint point lie within the radius of support of the point being evaluated).

\section{Structural analyses of cylindrical shells}

\section{Multiscale composite average properties}

In the present work, the reconstructed shells are made of laminated composite materials. Despite the development of a number of approaches to predict the macroscopic average properties of these materials, one can say that the rule of mixtures is one of the most usually used schemes to predict the average properties of such materials, mainly due to its simplicity. For this reason and because of the availability of published results based on this homogenization scheme, we will also use it here. In this study, one has additionally considered multiscale composites, which are obtained by the inclusion of carbon nanotubes (CNT) on the more "traditional" fibre reinforced laminated composites (Fig. 2).

This more recent composites constitution is leading to a new generation of advanced composite materials, in the field of advanced, high-performance materials (Raffie and He 2014). Nanotubes/fibre/polymer multiscale nanocomposites (CNTFPC) can reduce weight drastically with the same or better performance when compared to the traditional metallic materials and deserve a special attention. The nanocomposites can be regarded as isotropic materials, as the CNT are assumed to be uniformly dispersed and randomly oriented through the matrix. It is assumed that

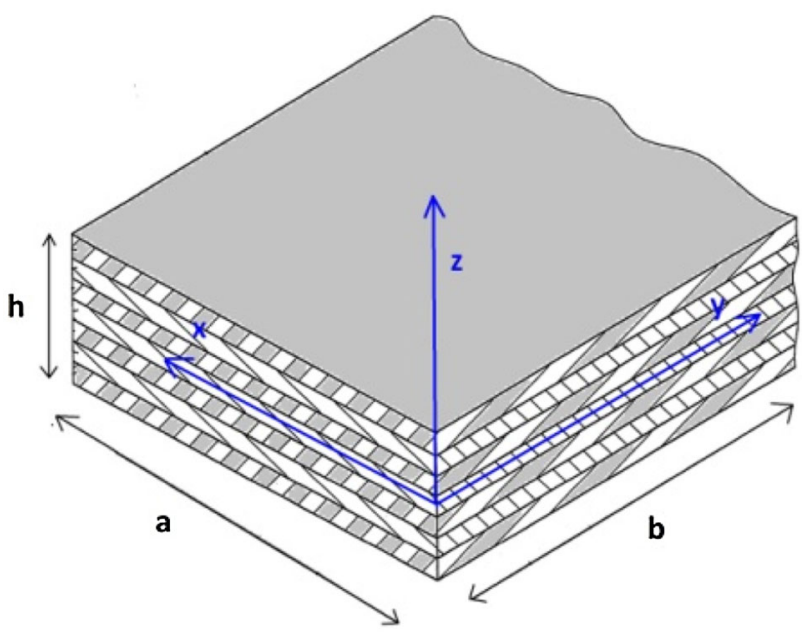

Fig. 2 Schematic representation multiscale composite laminate

these nanoinclusions show a perfect adhesion to the embedding matrix which is also considered to have no voids.

The average material properties of the multiscale laminated composite can be predicted according to a combination of Halpin-Tsai scheme and micromechanics approach via two steps in the hierarchy. Therefore, according to the Halpin-Tsai equation, the tensile modulus of the nanocomposite matrix, $E^{\mathrm{NC}}$, where the superscript $\mathrm{NC}$ stands for nanocomposite, can be expressed as follows:

$E^{\mathrm{NC}}=\frac{E^{\mathrm{ER}}}{8}\left[5\left(\frac{1+2 \beta_{d d} V^{\mathrm{CN}}}{1-\beta_{d d} V^{\mathrm{CN}}}\right)+3 \frac{1+2\left(l^{\mathrm{CN}} / d^{\mathrm{CN}}\right) \beta_{d l} V^{\mathrm{CN}}}{1-\beta_{d l} V^{\mathrm{CN}}}\right]$

with

$\beta_{d l}=\frac{\left(E_{11}^{\mathrm{CN}} / E^{\mathrm{ER}}\right)-\left(d^{\mathrm{CN}} \times 4 t^{\mathrm{CN}}\right)}{\left(E_{11}^{\mathrm{CN}} / E^{\mathrm{ER}}\right)+\left(l^{\mathrm{CN}} \times 2 t^{\mathrm{CN}}\right)}$
$\beta_{d d}=\frac{\left(E_{11}^{\mathrm{CN}} / E^{\mathrm{ER}}\right)-\left(d^{\mathrm{CN}} \times 4 t^{\mathrm{CN}}\right)}{\left(E_{11}^{\mathrm{CN}} / E^{\mathrm{ER}}\right)+\left(d^{\mathrm{CN}} \times 2 t^{\mathrm{CN}}\right)}$,

where $E_{11}^{\mathrm{CN}}, V^{\mathrm{CN}}, l^{\mathrm{CN}}, d^{\mathrm{CN}}$, and $t^{\mathrm{CN}}$ are the Young's modulus, volume fraction, length, outer diameter, and the thickness of CNT, respectively, and $V^{\mathrm{ER}}$ and $E^{\mathrm{ER}}$ indicate the volume fraction and Young's modulus of the isotropic epoxy resin matrix, respectively.

The volume fraction of CNT can be expressed as follows:

$V^{\mathrm{CN}}=\frac{w^{\mathrm{CN}}}{w^{\mathrm{CN}}+\left(\rho^{\mathrm{CN}} / \rho^{\mathrm{ER}}\right)-\left(\rho^{\mathrm{CN}} / \rho^{\mathrm{ER}}\right) w^{\mathrm{CN}}}$,

where $w^{\mathrm{CN}}$ is the weight fraction of the $\mathrm{CNT} ; \rho^{\mathrm{CN}}$ and $\rho^{\mathrm{ER}}$ are the densities of the CNT and epoxy resin matrix, respectively. The Poisson's ratio and mass density $\rho$ can be expressed using Voigt rule of mixtures: 
$v^{\mathrm{NC}}=V^{\mathrm{CN}} v^{\mathrm{CN}}+V^{\mathrm{ER}} v^{\mathrm{ER}}$

$\rho^{\mathrm{NC}}=V^{\mathrm{CN}} \rho^{\mathrm{CN}}+V^{\mathrm{ER}} \rho^{\mathrm{ER}}$

$V^{\mathrm{ER}}=1-V^{\mathrm{CN}}$

being $\rho^{\mathrm{CN}}$ and $\rho^{\mathrm{ER}}$ are the mass densities of the CNT and epoxy matrix, $v^{\mathrm{CN}}$ and $v^{\mathrm{ER}}$ are the Poisson's ratio of the CNT and the epoxy resin matrix, respectively. As the quantity of CNT dispersed in the epoxy resin is small, the Poisson's ratio of the CNT composite may be assumed to be the same as that of the epoxy resin. The weight fraction and consequently the volume fraction of the carbon nanotubes are considered to be sufficiently small not to compromise its dispersion in the matrix. This topic deserves a special attention, as from the studies carried out, one may conclude that a good dispersion of the carbon nanotubes is crucial to improve the mechanical response of a composite material. Nevertheless, obtaining a good dispersion is not easy, because, due to Van der Waals forces among the nanoinclusions and the surrounding matrix, the carbon nanotubes naturally tend to agglomerate and to constitute bundles or ropes. Although these agglomerates may contribute to a natural damping which may be useful in certain situations, their lower aspect ratios provide a more limited capacity to carry and transfer the loads ( $\mathrm{Hu}$ et al. 2006; Thostenson et al. 2005; Tornabene et al. 2016).

Finally, the average material properties of the final multiscale CNTFPC (carbon nanotubes/fibre/polymeric resin composite) are assumed to be orthotropic, and the determination of the elastic stiffness elastic coefficients is done using the corresponding material constitutive relation (Reddy 2004).

\section{Donnell first-order shear deformation theory}

To enable the analysis of the laminated composite shells, one considers the use of the first-order shear deformation theory (FSDT) of Donnell (Reddy 2004; Ferreira and Roque 2007, 2009). The shell is assumed to be constituted by a finite number of orthotropic layers with uniform thickness. One will consider that $\left(\xi_{1}, \xi_{2}, \zeta\right)$ denote the orthogonal curvilinear coordinates (shell coordinates), such that the $\xi_{1}$ - and $\xi_{2}$-curves are curvature lines on the middle surface $\zeta=0$, and $\zeta$-curves are straight lines orthogonal to the surface $\zeta=0$. For cylindrical and spherical shells, the lines of principal curvature coincide with the coordinate lines. The values of the principal radii of curvature are represented by $R_{1}$ and $R_{2}$. According to these assumptions, the displacement field is given as follows:

$$
\begin{aligned}
& u=\left(1+\frac{\zeta}{R_{1}}\right) u_{0}+\zeta \phi_{1} \\
& v=\left(1+\frac{\zeta}{R_{2}}\right) v_{0}+\zeta \phi_{2} \\
& w=w_{0},
\end{aligned}
$$

where $u_{0}$ and $v_{0}$ are the membrane displacements, $\phi_{1}$ and $\phi_{2}$ denote rotations of the normals relative to the $\xi_{1}$ and $\xi_{2}$ axes, respectively, and $w_{0}$ is the transverse deflection of the shell mid-plane. The relations among strains and displacements referred to an orthogonal curvilinear coordinate system lead to a deformation field described as follows, where $x$ and $y$ denote the cartesian coordinates:

$\epsilon=\left[\epsilon_{1}, \epsilon_{2}, \epsilon_{6}\right]^{\mathrm{T}}=(\epsilon 0+\zeta \mathrm{K}) ; \quad \epsilon_{\mathrm{S}}=\left[\epsilon_{4}, \epsilon_{5}\right]^{\mathrm{T}}$

being:

$$
\begin{aligned}
\epsilon 0 & =\left[u_{0, x}+w / R_{1}, v_{0, y}+w / R_{2}, u_{0, y}+v_{0, x}\right]^{\mathrm{T}} \\
K & =\left[\kappa_{1}, \kappa_{2}, \kappa_{6}\right]=\left[\phi_{1, x}, \phi_{2, y}, \phi_{1, y}+\phi_{2, x}\right]^{\mathrm{T}} \\
\epsilon_{\mathrm{S}} & =\left[\phi_{1}+w_{0, y}, \phi_{2}+w_{0, x}\right]^{\mathrm{T}}
\end{aligned}
$$

where $x$ and $y$ represent the Cartesian coordinates. The stress-strain relations for a generic $k$ th layer are then given as follows:

$$
\begin{aligned}
\sigma_{k} & =Q_{k} \epsilon k, \quad \tau_{k}=Q_{k}^{\mathrm{S}} \epsilon_{k}^{\mathrm{S}} \\
\sigma_{k} & =\left[\sigma_{1}, \sigma_{2}, \sigma_{6}\right]_{k}^{T}, \quad \epsilon=\left[\epsilon_{1}, \epsilon_{2}, \epsilon_{6}\right]_{k}^{\mathrm{T}} \\
\tau_{k} & =\left[\tau_{4}, \tau_{5}\right]_{k}^{\mathrm{T}}, \quad \epsilon_{k}^{\mathrm{S}}=\left[\epsilon_{4}, \epsilon_{5}\right]_{k}^{\mathrm{S}} .
\end{aligned}
$$

The expressions of the reduced stiffness elastic coefficients, as well as the transformed ones, are given in literature (Reddy 2004). The principle of virtual work for this problem yields:

$$
\begin{aligned}
& \int_{-h_{k} / 2}^{h_{k} / 2}\left(\int_{\Omega^{k}}\left[\sigma_{1} \delta \epsilon_{1}^{k}+\sigma_{2} \delta \epsilon_{2}^{k}+\sigma_{6} \delta \epsilon_{6}^{k}+\tau_{4} \delta \epsilon_{4}^{k}+\tau_{5} \delta \epsilon_{5}^{k}\right]\right) \\
& \mathrm{d} x \mathrm{~d} y \mathrm{~d} \zeta-\int_{\Omega}\left(q_{0} \delta w\right) \mathrm{d} x \mathrm{~d} y=0 .
\end{aligned}
$$

After integrating in the thickness, one obtains:

$$
\begin{gathered}
\int_{\Omega^{k}}\left[N_{1} \delta \epsilon 0_{1}+M_{1} \delta \kappa_{1}+N_{2} \delta \epsilon 0_{2}+M_{2} \delta \kappa_{2}+N_{6} \delta \epsilon 0_{6}\right. \\
\left.+M_{6} \delta \kappa_{6}+Q_{2} \delta \epsilon 0_{4}+Q_{1} \delta \epsilon 0_{5}-q_{0} \delta w\right] \mathrm{d} x \mathrm{~d} y=0
\end{gathered}
$$

being $q$ the distributed transverse load, and $N_{i}, M_{i}$, and $Q_{i}$ the stress resultants:

$$
\left(N_{i}, M_{i}\right)=\sum_{k=1}^{N} \int_{\zeta_{k}-1}^{\zeta_{k}} \sigma_{i}^{k}(1, \zeta) \mathrm{d} \zeta, \quad Q_{i}=\sum_{k=1}^{N} \int_{\zeta_{k}-1}^{\zeta_{k}} \tau_{i}^{k} \mathrm{~d} \zeta
$$

where $N$ denotes the number of the orthotropic layers of the shell and $\zeta_{k}, \zeta_{k-1}$ the top and bottom $\zeta$-coordinates of the $k$ th lamina. The governing equilibrium equations can be derived from Eq. 26, by integrating the displacement gradients by parts and setting the coefficients of $\delta u_{0}, \delta v_{0}, \delta w_{0}$, and $\delta \theta_{x, y}$ to zero (Ferreira and Roque 2007, 2009). 


\section{The Kansa method (multiquadrics)}

In this phase of the work, one considers multiquadric radial basis functions, which depend only on the distance to a centre point $x_{j}$ and has the form $\Phi\left(\| x-x_{i} \mid\right)$ (Kansa 1990). Thus, for a set of nodes $x_{j}, \ldots, x_{N} \in \Omega \subset \Re$, the radial basis functions centered at $x_{j}$ are defined as follows:

$\Phi_{j}(x) \equiv \Phi\left(\left\|x-x_{j}\right\|\right) \in \Re^{d}$,

where ||$x-x_{j} \mid$ is the Euclidean norm. The multiquadric functions used have the form: $\Phi=\sqrt{\left(\left(x-x_{j}\right)^{2}+c^{2}\right)}$ with $c=2 / \sqrt{N}$, in which $N$ denote the number of nodes used in each edge of the plate grid. One of the main advantages of radial basis functions is the insensitivity to spatial dimension, making the implementation of this method much easier than, e.g., finite elements. Pairwise distances between points are the only geometric properties required by the method.

Kansa's unsymmetrical collocation method is considered in the present work. Thus, a boundary-valued problem with a domain $\Omega \in \Re^{d}$ and a linear elliptic partial differential equation can be written as follows:

$$
\begin{aligned}
& \operatorname{Lu}(x)=s(x) \quad \text { in } \Omega \\
& \operatorname{Bu}(x)_{\delta \Omega}=s(x) \quad \text { on } \delta \Omega,
\end{aligned}
$$

where $\delta \Omega$ represents the boundary of the problem. We use points along the boundary $\left(x_{j}, j=1, \ldots, N_{\mathrm{B}}\right)$ and in the interior $\left(x_{j}, j=N_{\mathrm{B}}+1, \ldots, N\right)$. Collocation with the boundary data at the boundary points and with PDE ate the interior leads to equations:

$$
\begin{aligned}
& s_{\mathrm{B}}(x)=p_{m}(x)+\sum_{i^{n}} \lambda_{i} B \Phi\left(\left\|x-x_{j}\right\|\right)=F\left(x_{i}\right), \quad i=1, \ldots, N_{\mathrm{B}} \\
& s_{\mathrm{L}}(x)=p_{m}(x)+\sum_{i^{n}} \lambda_{i} L \Phi\left(\left\|x-x_{j}\right\|\right)=S\left(x_{i}\right), \quad i=N_{\mathrm{B}}+1, \ldots, N,
\end{aligned}
$$

with $F\left(x_{i}\right)$ and $S\left(x_{i}\right)$ being prescribed values at the boundary nodes and the function values at the interior nodes, respectively. This corresponds to a system of equations with an unsymmetrical coefficient matrix, which in matrix form appears as:

$$
\left[\begin{array}{l}
B \Phi \\
L \Phi
\end{array}\right][\lambda]=\left[\begin{array}{l}
F \\
S
\end{array}\right] .
$$

\section{Numerical applications}

In this section, we present two validation cases and a set of studies to illustrate the reconstruction of the shells and after that, their static analysis. To illustrate the present integrated methodology, we used synthetic point clouds, equivalent to what we would get if real clouds were used after removal of noisy points. The first set of points clouds represent cylindrical shells with $R / a=5, R / a=10, R / a$ $=20, R / a=50$ and $R / a=100$, where $R$ represent the curvature radius and $a$ is the shell side length. These clouds had, respectively, 436, 456, 471, 505, and 527 points, trying to denote a low resolution $3 \mathrm{D}$ laser scanning process. A similar methodology was followed for double-curvature shells, where the number of points was 529 in all the cases. Concerning to the static analysis of the shells, it was considered that they would have their edges, all simply supported or clamped, and the shape parameter c used, had an approximated value of $2 / \sqrt{\left(N_{\mathrm{B}} / 4\right)}$, for $N_{\mathrm{B}}$ total boundary points. This was because we found that the edges defined by the points provided by the isosurface approach were relatively regular.

\section{Validation examples}

\section{Laminated composite plates}

In the present case, two simply supported laminated plates with unit length edges are submitted to uniformly and sinusoidal distributed loads, respectively. The material properties of the first laminate, were: $E_{1}=E_{2}=10,920$, $G_{23}=G_{12}=G_{13}=E_{2} / 2.5$, and $v_{12}=v_{21}=0.25$, and for the second laminate: $E_{1}=10,920, \quad E_{2}=E_{1} / 25$ $G_{12}=G_{13}=0.5 E_{2}, G_{23}=0.2 E_{2}$, and $v_{12}=0.25$. The laminates possess the stacking sequence $\left[0^{\circ} / 90^{\circ} / 90^{\circ} / 0^{\circ}\right]$ with equal thickness layers.

In Table 1, we can observe the mid-plane maximum deflections obtained for the first laminate submitted to the uniform pressure, and the values provided by (Ferreira and Roque 2009) which used a third-order shear deformation theory (TSDT). The results are presented in a non-dimensional form using the multiplier $\bar{w}=10^{2} w_{\max } h^{3} / q_{0} a^{4}$.

In the second case, where a sinusoidal load $q_{z}=q_{0 \text { - }}$ $\sin (\pi x / a) \sin (\pi y / b)$ was applied, the results are also presented in a non-dimensional form in Table 2.

By observing the maximum transverse deflection values obtained, one can say that the present meshless approach performs extremely well in the case of using regular grids and the results obtained considering the randomly generated synthetic point cloud are also good for all the aspect ratios. It is important to mention the fact that the points considered and provided by the isosurface extraction in the reconstruction approach are randomly distributed in the space, giving additional uncontrolled parameters to the meshless method. 
Table 1 Non-dimensional transverse deflection $\bar{w}$

\begin{tabular}{lllccc}
\hline$a / h$ & $\begin{array}{l}\text { TSDT (Ferreira and } \\
\text { Roque 2009) }\end{array}$ & \multicolumn{2}{l}{ Present } & & \\
\cline { 3 - 6 } & & $17 \times 17$ grid & Dev $(\%)$ & Point cloud & Dev (\%) \\
\hline 10 & 4.7764 & 4.7683 & 0.17 & 4.7070 & 1.45 \\
20 & 4.6077 & 4.6066 & 0.02 & 4.5391 & 1.49 \\
50 & 4.5671 & 4.5749 & -0.17 & 4.4777 & 1.96 \\
100 & 4.5391 & 4.5838 & -0.98 & 4.4986 & 0.89 \\
\hline
\end{tabular}

First laminate

\begin{tabular}{llllll}
\hline$a / h$ & $\begin{array}{l}\text { TSDT (Ferreira and } \\
\text { Roque 2009) }\end{array}$ & Present & & \\
\cline { 3 - 5 } & & $17 \times 17$ grid & Dev (\%) & Point cloud & Dev (\%) \\
\hline 4 & 1.7100 & 1.7087 & 0.08 & 1.6855 & 1.43 \\
10 & 0.6628 & 0.6621 & 0.11 & 0.6477 & 2.28 \\
20 & 0.4912 & 0.4908 & 0.08 & 0.4829 & 1.69 \\
100 & 0.4337 & 0.4375 & -0.88 & 0.4288 & 1.13 \\
\hline
\end{tabular}

Table 2 Non-dimensional transverse deflection $\bar{w}$. Second laminate

\section{Laminated composite shells}

In this case, it is considered a simply supported laminated shell with an aspect ratio $a / h=10$, submitted to a uniformly distributed load. The material properties of the first laminate considered in this validation case were the following: $E_{1}=10,920, E_{2}=\frac{E_{1}}{25}, G_{12}=G_{13}=0.5 E_{2}, G_{23}=$ $0.2 E_{2}, v_{12}=0.25$ being considered the layered configuration $\left[90^{\circ} / 0^{\circ}\right]$. In Table 3 , we can observe the mid-plane non-dimensional maximum deflections obtained with the present model and the reference results (Reddy 2004). One has used the same multiplier.

These results are complemented with the ones shown in Table 4, obtained by Reddy (2004), where an additional stacking sequence $\left[0^{\circ} / 90^{\circ} / 0^{\circ}\right]$ is also available. From the results obtained, it is possible to conclude that the approach used in the present work is also suitable for the static analysis of moderately thick plates.

In the cases of unstructured point-clouds, the deviations are higher but continue to be very acceptable, especially if we refer the fact that the multiquadric method dependency of the shape parameter needs to be further addressed.

In fact, when a regular grid is considered, a value of $2 / \sqrt{(N)}$, where $N$ denotes the number of nodes used in each edge of the plate grid, is appropriate. In more uncontrolled situations, according to the studies carried out, one can say that this subject may be object of further studies, although there already exists, some optimization approaches performed to find the optimum value of the shape parameter.

\section{Case study: cylindrical shells}

\section{Reconstruction of cylindrical shells}

In this subsection, one presents the results obtained considering the reconstruction of cylindrical shells. For illustration purposes, in Figs. 3 and 4, we can observe, respectively, the original point cloud from which the surfaces of the cylindrical shell with $R_{1 / a}=5$ were reconstructed and the associated reconstructed surface.

The algorithm here implemented to reconstruct the surfaces has a set of input parameters which have shown to have a significant influence in the quality of the reconstructed surface (Bernardo and Loja 2015). Hence, one can say that the most relevant parameters in the algorithm are $N_{\text {eval }}, N_{\text {cell }}, R, R_{\text {no, }}$, and the normal criterion. Briefly, describing those parameters, we can refer to the first as the number of edge points of the 3D grid used to evaluate the interpolated RBF function.
Table 3 Non-dimensional transverse deflection $\bar{w}$

\begin{tabular}{lcllrr}
\hline$a / h$ & FSDT (Reddy 2004) & Present & & \\
\cline { 3 - 5 } & & $17 \times 17$ grid & Dev $(\%)$ & Point cloud & Dev (\%) \\
\hline 5 & 9.8249 & 9.8058 & 0.19 & 9.9513 & -1.29 \\
10 & 10.1410 & 10.1018 & 0.39 & 10.1249 & 0.16 \\
\hline
\end{tabular}

Laminated shell $\left[0^{\circ} / 90^{\circ}\right]$ 
Table 4 Non-dimensional transverse deflection $\bar{w}$

\begin{tabular}{|c|c|c|c|c|c|c|c|}
\hline \multirow[t]{2}{*}{ Stack } & \multirow[t]{2}{*}{$a / h$} & \multirow[t]{2}{*}{$R / a$} & \multirow[t]{2}{*}{ FSDT (Reddy 2004) } & \multicolumn{4}{|l|}{ Present } \\
\hline & & & & $25 \times 25$ grid & $\operatorname{Dev}(\%)$ & Point cloud & $\operatorname{Dev}(\%)$ \\
\hline \multirow[t]{6}{*}[0^{\circ}/90^{\circ}]{} & \multirow[t]{3}{*}{10} & 5 & 17.9440 & 18.1077 & -0.91 & 18.2555 & -1.74 \\
\hline & & 10 & 19.0650 & 19.1158 & -0.27 & 19.1291 & -0.34 \\
\hline & & $10^{30}$ & 19.4690 & 19.4422 & 0.14 & 19.5235 & -0.28 \\
\hline & \multirow[t]{3}{*}{100} & 5 & 1.7535 & 1.7646 & -0.63 & 1.7044 & 2.80 \\
\hline & & 10 & 5.5428 & 5.5359 & 0.12 & 5.6043 & -1.11 \\
\hline & & $10^{30}$ & 16.9800 & 16.9939 & -0.08 & 16.7319 & 1.46 \\
\hline \multirow[t]{6}{*}[0^{\circ}/90^{\circ}/0^{\circ}]{} & \multirow[t]{3}{*}{10} & 5 & 9.7937 & 10.0367 & -2.48 & 9.6084 & 1.89 \\
\hline & & 10 & 10.1100 & 10.0125 & 0.96 & 10.2423 & -1.31 \\
\hline & & $10^{30}$ & 10.2200 & 10.2124 & 0.07 & 10.0574 & 1.59 \\
\hline & \multirow[t]{3}{*}{100} & 5 & 1.5118 & 1.4888 & 2.27 & 1.4663 & 3.01 \\
\hline & & 10 & 3.6445 & 3.6068 & 1.03 & 3.5823 & 1.71 \\
\hline & & $10^{30}$ & 6.6970 & 6.6889 & 0.12 & 6.5829 & 1.70 \\
\hline
\end{tabular}

Laminated shell $\left[0^{\circ} / 90^{\circ}\right],\left[0^{\circ} / 90^{\circ} / 0^{\circ}\right]$

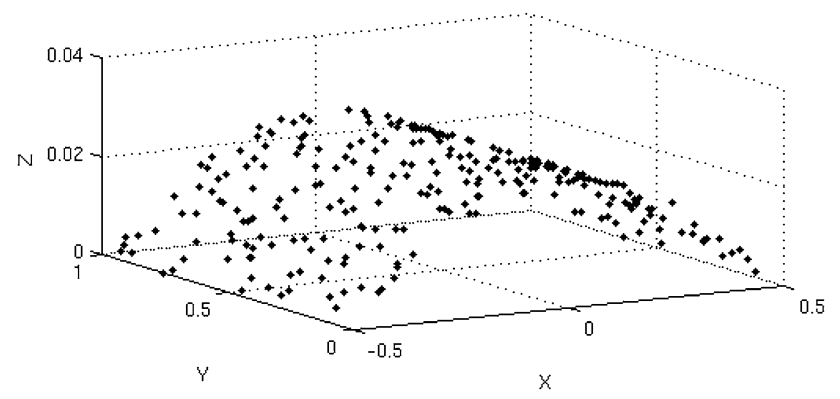

Fig. 3 Synthetic point cloud of the cylindrical shell with $R_{1 / a}=5$, $a=1,436$ points

The second denotes the number of edge points of the 3D grid used as centres of the support domain when the algorithm interpolates and evaluates the functions in each iteration. The support radius $R$ and $R_{\text {no }}$ are the radii used to search for the neighbours (making use of the $k d$ tree) during the interpolation phase and to search for the neighbours that will be used to compute the normal vectors of each point of the point, which is considered if the scalar product $n_{i} \cdot n_{j}$ (only for closest neighbours) lay in the user established interval. Such interval denotes the normal criterion.

In addition, one can say that, in all the cases, we used the following values for such parameters: $N_{\text {eval }}=15$, $N_{\text {cell }}=10, R=0.25, R_{\mathrm{no}}=0.175$ and the normal criterion in the interval [0.98-1.02]. From the previous studies carried out, one can conclude that these values are appropriate for the reconstruction of the shell surfaces. We also considered the compactly supported function CS-RBF C6 in all cases.

Detailed information about these parameters is presented in (Bernardo and Loja 2015). In addition, one must say that the isosurface extraction carried out to reconstruct the surfaces, provided 236, 236, 315, 259, and 257 points in the cases where $R_{1 / a}=5, R_{1 / a}=10, R_{1 / a}=20, R_{1 / a}=50$, and $R_{1 / a}=100$, respectively.

In Fig. 4, one presents the extracted isosurface points (in red) lying in the reconstructed surface. By observing it, one can conclude that the present CS-RBF approach used to reconstruct the shell surface provides a good approximation of the surface represented by the randomly distributed points shown in Fig. 3. This turns expectable a relevant
Fig. 4 Left reconstructed surface of the cylindrical shell, and the points (in red, 236) provided by the isosurface extraction. Right top view of the surface

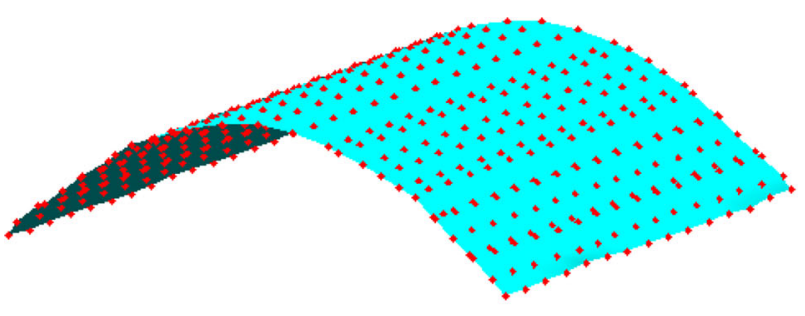


Table 5 Cases considered in the static analyses of cylindrical shells

\begin{tabular}{llllll}
\hline Case & $E_{\mathrm{M}}(\mathrm{GPa})$ & $v_{\mathrm{M}}$ & $E_{\mathrm{F}}(\mathrm{GPa})$ & $v_{\mathrm{F}}$ & Other parameters \\
\hline 1 & 2.72 & 0.3 & 69 & 0.2 & {$\left[00^{\circ} / 90^{\circ} / 0^{\circ}\right]$} \\
2 & Single-walled CNT & & & & {$\left[0 \% 90^{\circ}\right], R_{1 / a}=R_{2 / a}=\inf$} \\
& 11.15 & & & & \\
3 & Multi-walled CNT & & & & \\
& 3.19 & & & & \\
\hline
\end{tabular}

level of confidence in the final output which will be used in the next step of static analysis.

\section{Static analysis of cylindrical shells}

In the previous subsection, we performed the reconstruction of several cylindrical shells surfaces, being shown as an example the mid-surface of a shell with a radii/length $R_{1 / a}=5$. In the present subsection, we are considering that the reconstructed surfaces using the points obtained in the isosurface extraction, which after imposing the boundary conditions and applying a uniformly distributed load, are going to be submitted to a static analysis study.

The material and geometrical properties are shown in Table 5, in which the second and third cases consider nanotubes (CNT) inclusions with a weight fraction contents $w^{\mathrm{CN}}=0.04$, and in all of them, the edges are simply supported. A uniformly distributed load $p_{0}=10^{5}$ is applied. The other materials considered for the matrix and reinforcement phases were, respectively, epoxy resin and E-glass.

In all the cases, one has performed the calculation of the deflections using a regular mesh grid and a unstructured point cloud in other to compare the deviations between situations where the parameters are controlled and where it this does not occur. Tables $6,7,8$ show the results of the deflections obtained for the three distinct cases.

From Tables 6 and 7, one can conclude that the results obtained when we consider a point-cloud are close to those obtained in more controlled situations, especially if we refer to shells with smaller curvatures. In fact, as expected with the increase of the curvature, the deviation also gets higher, although acceptable. This is due to the fact that, in such situations, the shell is deeper and the first-order shear deformation theory may give these deviations.

In addition, from these tables, one can refer that in the case of $\left[0^{\circ} / 90^{\circ} / 0^{\circ}\right]$, the deviations are higher than those obtained in $\left[0^{\circ} / 90^{\circ}\right]$ configuration.

In the second case, the composite plate has a stacking sequence $\left[0^{\circ} / 90^{\circ}\right]$ and a 0.8 volume fraction is considered. The results obtained are presented in Table 8, for two aspect ratios, $a / h=10$ and $a / h=20$ allow concluding on a fair agreement for all the aspect ratios.
Table 6 First case maximum deflections $w\left(10^{-4} \mathrm{~m}\right)\left(\left[0^{\circ} / 90^{\circ} / 0^{\circ}\right]\right.$; $\left.V_{\mathrm{f}}=0.8, a / h=10\right)$

\begin{tabular}{lrllc}
\hline Stacking sequence & $R_{1 / a}$ & $17 \times 17$ grid & Point cloud & Dev $(\%)$ \\
\hline$\left[0^{\circ} / 90^{\circ}\right]$ & 5 & 3.0669 & 2.9193 & 4.81 \\
& 20 & 3.0488 & 3.0263 & 0.74 \\
{$\left[0^{\circ} / 90^{\circ} / 0^{\circ}\right]$} & 100 & 3.0532 & 3.0466 & 0.22 \\
& 5 & 2.2326 & 2.3669 & -6.02 \\
& 20 & 2.1342 & 2.1784 & -2.07 \\
& 100 & 2.0749 & 2.0991 & -1.17 \\
\hline
\end{tabular}

Table 7 First case-maximum deflections $w\left(10^{-3} \mathrm{~m}\right)\left(V_{\mathrm{f}}=0.8, a /\right.$ $h=20$ )

\begin{tabular}{lrlll}
\hline Stacking sequence & $R_{1 / a}$ & $17 \times 17$ grid & Point cloud & Dev $(\%)$ \\
\hline$\left[0^{\circ} / 90^{\circ}\right]$ & 5 & 2.2560 & 2.1164 & 6.19 \\
& 20 & 2.3393 & 2.3204 & 0.81 \\
{$\left[0^{\circ} / 90^{\circ} / 0^{\circ}\right]$} & 100 & 2.3295 & 2.9089 & 0.88 \\
& 5 & 1.3548 & 1.3330 & 1.61 \\
& 20 & 1.4100 & 1.3762 & 2.40 \\
& 100 & 1.6228 & 1.5850 & 2.33 \\
\hline
\end{tabular}

By observing Table 9, it can be seen that the results obtained when we consider a point-cloud are close to those obtained using a regular grid of points, for the different aspect ratios.

Tables 9, 10 are related to the maximum transverse displacements of the cylindrical shells with different aspect ratios $a / h$ and $R_{1 / a}$, with single-walled and multi-walled carbon nanotubes. There are also considered two different stacking sequences $\left[0^{\circ} / 90^{\circ}\right]$ and $\left[0^{\circ} / 90^{\circ} / 0^{\circ}\right]$.

From these results, it is possible to understand from a similar trend between the different stacking sequences situations. It is also possible to see that the single-walled nanotubes provide a stiffer behaviour when compared to the multi-walled ones. This is an expected situation considering its material properties, particularly the aspect ratio of the multi-walled carbon nanotubes (Costa and Loja 2016). Once the deflections, as well as the rotations around $x$ and $y$-axes are obtained, the reconstruction of these generalized displacements' surfaces along the unitary length shell is easily carried out. In Fig. 5, one illustrates 
Table 8 Second casemaximum deflections $w(\mathrm{~m})$

\begin{tabular}{llllllll}
\hline alh & \multicolumn{2}{l}{ Single-walled CNT } & & \multicolumn{3}{l}{ Multi-walled CNT } \\
\cline { 2 - 3 } & $17 \times 17$ grid & Point cloud & Dev $(\%)$ & & $17 \times 17$ grid & Point cloud & Dev $(\%)$ \\
\hline 10 & $1.8854 E-4$ & $1.8617 E-4$ & 1.26 & & $4.5818 E-4$ & $4.5242 E-4$ & 1.26 \\
20 & $1.4486 E-3$ & $1.4271 E-3$ & 1.48 & & $3.4808 E-3$ & $3.4400 E-3$ & 1.17 \\
50 & $2.2447 E-2$ & $2.2191 E-2$ & 1.14 & & $5.3858 E-2$ & $5.3011 E-2$ & 1.57 \\
100 & $1.8011 E-1$ & $1.7639 E-1$ & 2.07 & & $4.3587 E-1$ & $4.2394 E-1$ & 2.74 \\
\hline
\end{tabular}

$\left[0^{\circ} / 90^{\circ}\right]$ plate

Table 9 Third case-maximum deflections $w\left(10^{-4} \mathrm{~m}\right)(a / h=10)$

\begin{tabular}{|c|c|c|c|c|c|c|c|}
\hline \multirow[t]{2}{*}{ Stacking sequence } & \multirow[t]{2}{*}{$R_{1 / a}$} & \multicolumn{3}{|c|}{ Single-walled CNT } & \multicolumn{3}{|c|}{ Multi-walled CNT } \\
\hline & & $17 \times 17$ grid & Point cloud & $\operatorname{Dev}(\%)$ & $17 \times 17$ grid & Point cloud & $\operatorname{Dev}(\%)$ \\
\hline \multirow[t]{3}{*}[0^{\circ}/90^{\circ}]{} & 5 & 1.8479 & 1.8945 & -2.52 & 4.5365 & 4.5216 & 0.33 \\
\hline & 20 & 1.8842 & 1.8508 & 1.77 & 4.5763 & 4.5085 & 1.48 \\
\hline & 100 & 1.8863 & 1.8764 & 0.52 & 4.5821 & 4.5523 & 0.65 \\
\hline \multirow[t]{3}{*}[0^{\circ}/90^{\circ}/0^{\circ}]{} & 5 & 1.8246 & 1.8757 & -2.80 & 3.2342 & 3.3100 & -2.34 \\
\hline & 20 & 2.0938 & 2.1462 & -2.50 & 2.8205 & 2.8644 & -1.56 \\
\hline & 100 & 1.2987 & 1.3102 & -0.89 & 2.9313 & 2.9012 & 1.03 \\
\hline
\end{tabular}

Table 10 Third case-maximum deflections $w\left(10^{-3} \mathrm{~m}\right)(a / h=20)$

\begin{tabular}{|c|c|c|c|c|c|c|c|}
\hline \multirow[t]{2}{*}{ Stacking sequence } & \multirow[t]{2}{*}{$R_{1 / a}$} & \multicolumn{3}{|c|}{ Single-walled CNT } & \multicolumn{3}{|c|}{ Multi-walled CNT } \\
\hline & & $17 \times 17$ grid & Point cloud & $\operatorname{Dev}(\%)$ & $17 \times 17$ grid & Point cloud & $\operatorname{Dev}(\%)$ \\
\hline \multirow[t]{3}{*}[0^{\circ}/90^{\circ}]{} & 5 & 1.3929 & 1.4466 & -3.86 & 3.3965 & 3.5010 & -3.08 \\
\hline & 20 & 1.4458 & 1.4241 & 1.50 & 3.5162 & 3.4486 & 1.92 \\
\hline & 100 & 1.4329 & 1.4400 & -0.50 & 3.4799 & 3.4545 & 0.73 \\
\hline \multirow[t]{3}{*}[0^{\circ}/90^{\circ}/0^{\circ}]{} & 5 & 1.3348 & 1.3801 & -3.39 & 1.8788 & 1.9143 & -1.89 \\
\hline & 20 & 2.2829 & 2.3520 & -3.03 & 2.1497 & 2.1667 & -0.79 \\
\hline & 100 & 1.1915 & 1.2111 & -1.64 & 1.9522 & 1.9755 & -1.19 \\
\hline
\end{tabular}
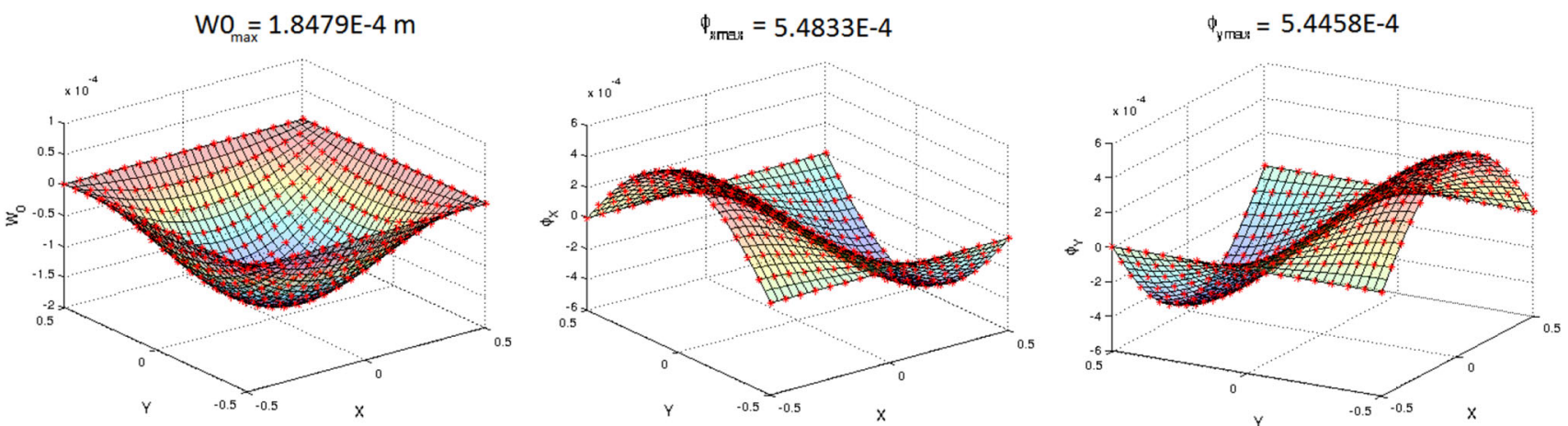

Fig. 5 Deflections (left), rotations around $x$-axis (centre) and $y$-axis (right) reconstruction from point cloud of the cylindrical shell with $R_{1 / a}=5$

these distributions along the shell domain, considering a ratio $R_{1 / a}=5$, a fibre volume fraction $V_{\mathrm{F}}=0.6$, with the inclusion of single-walled nanotubes in the matrix, a stacking sequence of $[0 / 90]$ and an aspect ratio $a / h=10$.
These generalized displacements distribution surfaces, correspond, respectively, to the transverse deflection and the rotations degrees of freedom (see displacement field) which were reconstructed using the 
same method implemented for the surfaces geometrical reconstruction.

\section{Case study: double-curvature shells}

\section{Reconstruction of double-curvature shells}

In this subsection, one presents the results obtained considering the reconstruction of double-curvature shells. For illustrating purposes, in Figs. 6 and 7, we can observe, respectively, the synthetic point cloud randomly generated for a subsequent reconstruction the surfaces of a double-

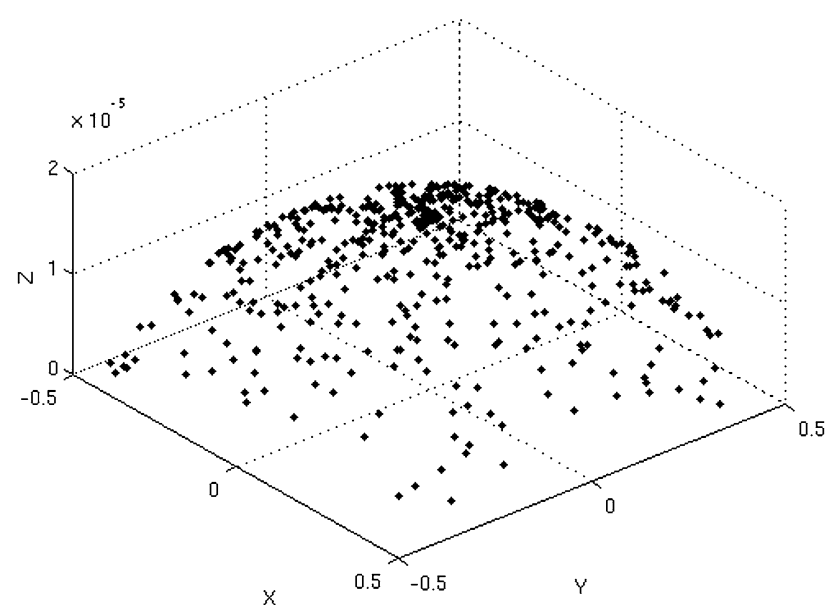

Fig. 6 Synthetic point cloud of the doubled-curved shell with $R_{1 / a}=5$

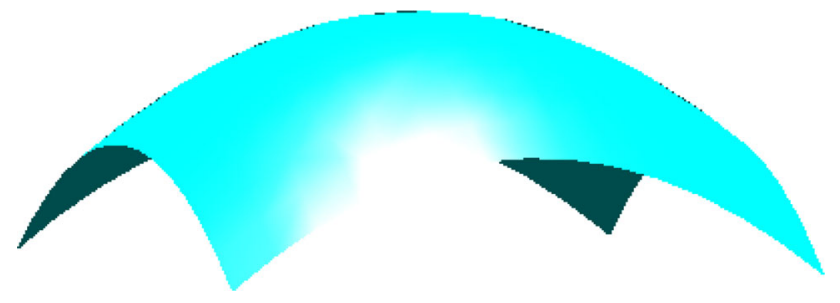

Fig. 7 Surface reconstruction from point cloud of the doubled-curved shell with $R_{1 / a}=5$ curvature shell with $R_{1 / a}=R_{2 / a}=5$ with a unitary edge length, and the associated reconstructed surface. The original synthetic point cloud had 529 points and the isosurface points considered were 277 .

The remaining parameters used in the reconstruction of the shells' surfaces were the same as already considered in previous cases.

From Figs. 7 and 8, it is possible to conclude on a good quality of the results in the reconstruction phase, being visible in Fig. 8, the representation of the surface, and the super-imposition of the isosurface points (in red), where it is visible a good fitting.

\section{Static analysis of double-curvature shells}

The present subsection considers the reconstructed surfaces using the points obtained in the isosurface extraction and, after imposing the boundary conditions, the static analysis was carried out for the four distinct cases enumerated in Table 11. There were applied two distinct types of unitary loads: a uniformly distributed load or a sinusoidal load.

In all the cases, the deflections were obtained using a regular mesh grid and the unstructured point cloud. In all the cases, where CNT were used, a weight fraction of $4 \%$ was considered.

Tables 12, 13, 14, 15, 16 present the results obtained for these cases, considering double-curvature shells with $R_{1}=R_{2}$.

From Table 10 and partially from Table 14, where we can compare the deflections obtained using a regular grid and the unstructured point-cloud, we can conclude that the deviations calculated are similar to those obtained in the $\left[0^{\circ} / 90^{\circ}\right]$ cylindrical shells.

The cases with a stacking sequence of $\left[0^{\circ} / 90 \% 0^{\circ}\right]$ seem to follow the same trend, being the deviations higher, although acceptable, in the cases where we have deeper shells $(R / a=5)$, which is expected considering the shear deformation theory used in this study.

Furthermore, one can say that, as expected, for the same load, geometrical characteristics, and materials, the
Fig. 8 Surface reconstruction from point cloud of the doubledcurved shell with $R_{1 / a}=5$
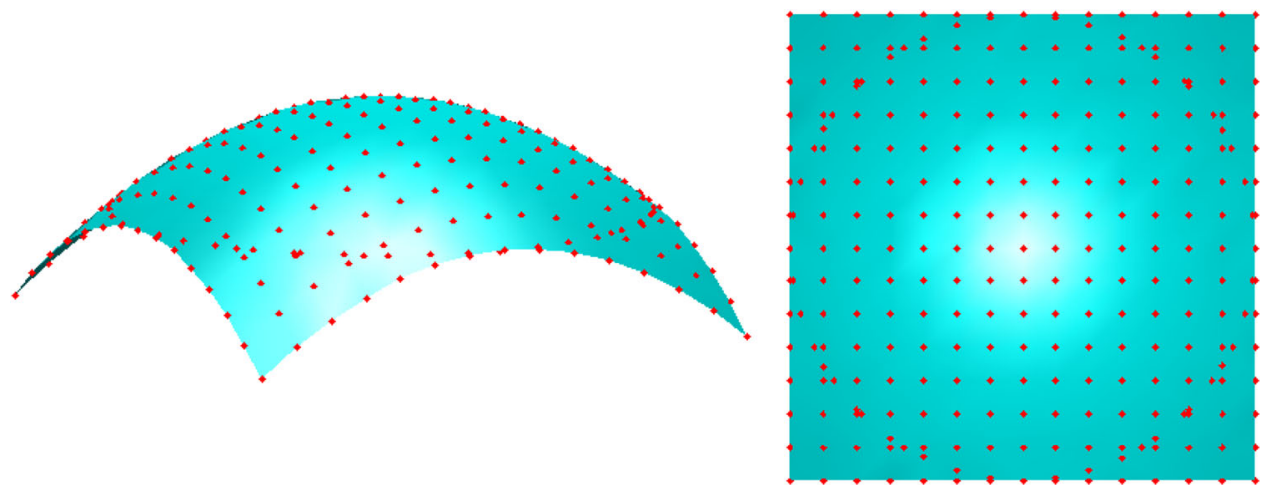
Table 11 Cases considered in the static analyses of doublecurvature shells

\begin{tabular}{llllll}
\hline Case & $E_{\mathrm{M}}(\mathrm{GPa})$ & $v_{\mathrm{M}}$ & $E_{\mathrm{F}}(\mathrm{GPa})$ & $v_{\mathrm{F}}$ & Other conditions \\
\hline 4 & Single-walled CNT: 11.15 & 0.3 & 69 & 0.2 & {$\left[0^{\circ} / 90^{\circ}\right]$} \\
& Multi-walled CNT: 3.19 & & & Clamped edges \\
5 & Without CNT: 2.72 & & {$\left[0^{\circ} / 90^{\circ} / 90^{\circ} / 0^{\circ}\right]$} \\
6 & Single-walled CNT: 11.15 & & & Sinusoidal load $p_{0}=10^{5}$ \\
7 & Multi-walled CNT: 3.19 & & & \\
\hline
\end{tabular}

Table 12 Fourth case-maximum deflections $\left(V_{\mathrm{f}}=0.6\right)$

\begin{tabular}{|c|c|c|c|c|c|c|c|c|c|c|}
\hline \multirow[t]{2}{*}{$a / h$} & \multirow[t]{2}{*}{$R / a$} & \multicolumn{3}{|c|}{ Single-walled CNT } & \multicolumn{3}{|c|}{ Multi-walled CNT } & \multicolumn{3}{|l|}{ Without CNT } \\
\hline & & $17 \times 17$ grid & Point cloud & $\operatorname{Dev}(\%)$ & $17 \times 17$ grid & Point cloud & $\operatorname{Dev}(\%)$ & $17 \times 17$ grid & Point cloud & $\operatorname{Dev}(\%)$ \\
\hline \multirow[t]{3}{*}{10} & 5 & $5.924 E-E-5$ & $6.124 E-5$ & -3.37 & $1.238 E-4$ & $1.265 E-4$ & -2.21 & $1.380 E-4$ & $1.353 E-4$ & 1.98 \\
\hline & 20 & $6.155 E-5$ & $6.283 E-5$ & -2.08 & $1.410 E-4$ & $1.421 E-4$ & -0.83 & $1.562 E-4$ & $1.583 E-4$ & -1.35 \\
\hline & 100 & $6.178 E-5$ & $6.199 E-5$ & -0.33 & $1.435 E-4$ & $1.430 E-4$ & 0.38 & $1.592 E-4$ & $1.592 E-4$ & -0.05 \\
\hline \multirow[t]{3}{*}{20} & 5 & $3.538 E-E-4$ & $3.606 E-4$ & -1.91 & $6.500 E-4$ & $6.526 E-4$ & 0.37 & $6.788 E-4$ & $6.686 E-4$ & 1.50 \\
\hline & 20 & $4.260 E-4$ & $4.222 E-4$ & 0.88 & $9.254 E-4$ & $9.158 E-4$ & 1.03 & $9.465 E-4$ & $9.378 E-4$ & 0.92 \\
\hline & 100 & $4.286 E-4$ & $4.293 E-4$ & -0.17 & $9.452 E-4$ & $9.492 E-4$ & -0.43 & $1.042 E-4$ & $1.052 E-3$ & -1.00 \\
\hline \multirow[t]{3}{*}{50} & 5 & $2.120 E-3$ & $2.071 E-3$ & 2.29 & $2.457 E-3$ & $2.409 E-3$ & 1.96 & $3.624 E-3$ & $3.587 E-3$ & 1.04 \\
\hline & 20 & $4.669 E-3$ & $4.676 E-3$ & -0.95 & $1.075 E-2$ & $1.054 E-2$ & 1.91 & $1.222 E-2$ & $1.241 E-2$ & -1.55 \\
\hline & 100 & $6.797 E-3$ & $6.754 E-3$ & 0.64 & $1.325 E-2$ & $1.305 E-2$ & 1.48 & $1.526 E-2$ & $1.535 E-2$ & -0.60 \\
\hline
\end{tabular}

Clamped edges

Table 13 Fifth case-maximum deflections $\left(V_{\mathrm{f}}=0.6\right)$

\begin{tabular}{|c|c|c|c|c|c|c|c|c|c|c|}
\hline \multirow[t]{2}{*}{$a / h$} & \multirow[t]{2}{*}{$R / a$} & \multicolumn{3}{|c|}{ Single-walled CNT } & \multicolumn{3}{|c|}{ Multi-walled CNT } & \multicolumn{3}{|c|}{ Without nanotubes } \\
\hline & & $17 \times 17$ grid & Point cloud & $\operatorname{Dev}(\%)$ & $17 \times 17$ grid & Point cloud & $\operatorname{Dev}(\%)$ & $17 \times 17$ grid & Point cloud & $\operatorname{Dev}(\%)$ \\
\hline \multirow[t]{3}{*}{10} & 5 & $1.275 E-4$ & $1.303 E-4$ & -2.13 & $1.619 E-4$ & $1.662 E-4$ & -2.69 & $2.682 E-4$ & $2.740 E-4$ & -2.15 \\
\hline & 20 & $1.404 E-4$ & $1.421 E-4$ & -1.24 & $2.496 E-4$ & $2.528 E-4$ & -1.27 & $3.114 E-4$ & $3.039 E-4$ & 2.42 \\
\hline & 100 & $1.499 E-4$ & $1.487 E-4$ & 0.77 & $2.565 E-4$ & $2.588 E-4$ & -0.89 & $3.390 E-4$ & $3.411 E-4$ & -0.63 \\
\hline \multirow[t]{3}{*}{20} & 5 & $8.069 E-4$ & $8.160 E-4$ & -1.13 & $1.361 E-3$ & $1.369 E-3$ & -0.59 & $1.685 E-3$ & $1.722 E-3$ & -2.20 \\
\hline & 20 & $1.122 E-3$ & $1.112 E-3$ & 0.91 & $2.299 E-3$ & $2.312 E-3$ & -0.56 & $2.386 E-3$ & $2.399 E-3$ & -0.53 \\
\hline & 100 & $1.241 E-3$ & $1.255 E-3$ & -1.11 & $2.557 E-E-3$ & $2.574 E-3$ & -0.68 & $2.686 E-3$ & $2.656 E-3$ & 1.12 \\
\hline \multirow[t]{3}{*}{50} & 5 & $4.742 E-3$ & $4.850 E-3$ & -2.28 & $5.338 E-3$ & $5.267 E-3$ & 1.33 & $1.663 E-2$ & $1.687 E-2$ & -1.42 \\
\hline & 20 & $7.883 E-3$ & $7.912 E-3$ & -0.37 & $1.757 E-2$ & $1.723 E-2$ & 1.96 & $2.776 E-2$ & $2.740 E-2$ & -1.31 \\
\hline & 100 & $9.570 E-3$ & $9.455 E-3$ & 1.20 & $2.257 E-2$ & $2.266 E-2$ & -0.42 & $3.377 E-2$ & $3.388 E-2$ & -0.32 \\
\hline
\end{tabular}

maximum deflections are smaller when the edges are clamped, which is an expected response pattern.

Table 13 confirms that the present approach leads again to good results, with low deviations, in a wide range of aspect ratios, from moderately thick to thin double-curvature laminated shells, with diverse stacking sequences, under different boundary conditions.

Again, from comparing the deflections obtained using a regular grid and a point-cloud, shown in Tables 12 and 13, we can withdraw similar conclusions to the previous cases.
For the present case study, Fig. 9 shows the shell deflections, and rotations around $x$-axis and around $y$-axis of a regular grid shell. In this illustration, it was considered the case with the following parameters $a / h=10, R /$ $a=20, V_{\mathrm{F}}=0.6, w^{\mathrm{CN}}=0.04$, and the stacking sequence $\left[0^{\circ} / 90^{\circ}\right]$, being all the edges clamped. The shell was subjected to a uniformly distributed load.

The mentioned degrees of freedom surfaces of the shell were reconstructed using the same method developed and implemented for surfaces reconstructions departing from unstructured points' clouds. 
Table 14 Sixth case-maximum deflections $\left(V_{\mathrm{f}}=0.6\right)$

\begin{tabular}{|c|c|c|c|c|c|c|c|c|}
\hline \multirow[t]{2}{*}{ Stacking sequence } & \multirow[t]{2}{*}{$a / h$} & \multirow[t]{2}{*}{$R / a$} & \multicolumn{3}{|c|}{ Single-walled CNT } & \multicolumn{3}{|c|}{ Multi-walled CNT } \\
\hline & & & $17 \times 17$ grid & Point cloud & $\operatorname{Dev}(\%)$ & $17 \times 17$ grid & Point cloud & $\operatorname{Dev}(\%)$ \\
\hline \multirow[t]{9}{*}[0^{\circ}/90^{\circ}]{} & \multirow[t]{3}{*}{$10\left(10^{-4} w\right)$} & 5 & 1.0951 & 1.0834 & 1.07 & 2.6726 & 2.7318 & -2.22 \\
\hline & & 20 & 1.1927 & 1.1851 & 0.64 & 2.8960 & 2.8987 & -0.09 \\
\hline & & 100 & 1.1991 & 1.2010 & -0.16 & 2.9119 & 2.8969 & 0.52 \\
\hline & \multirow[t]{3}{*}{$20\left(10^{-3} w\right)$} & 5 & 0.6835 & 0.6675 & 2.34 & 1.6540 & 1.6451 & 0.54 \\
\hline & & 20 & 0.9015 & 0.8892 & 1.37 & 2.1619 & 2.1260 & 1.66 \\
\hline & & 100 & 0.9201 & 0.9171 & 0.33 & 2.2053 & 2.2220 & -0.76 \\
\hline & \multirow[t]{3}{*}{$50\left(10^{-2} w\right)$} & 5 & 0.4763 & 0.4714 & 1.04 & 1.1291 & 1.1029 & 2.32 \\
\hline & & 20 & 1.2708 & 1.2710 & -0.02 & 3.0455 & 3.0192 & 0.86 \\
\hline & & 100 & 1.4277 & 1.4174 & 0.72 & 3.4094 & 3.4396 & -0.89 \\
\hline \multirow[t]{9}{*}[0^{\circ}/90^{\circ}/0^{\circ}]{} & \multirow[t]{3}{*}{$10\left(10^{-4} w\right)$} & 5 & 1.0363 & 1.0690 & -3.16 & 1.9700 & 2.0287 & -2.98 \\
\hline & & 20 & 1.1168 & 1.1255 & -0.78 & 2.0984 & 2.0505 & 2.28 \\
\hline & & 100 & 1.1735 & 1.1438 & 2.53 & 2.6213 & 2.5800 & 1.58 \\
\hline & \multirow[t]{3}{*}{$20\left(10^{-3} w\right)$} & 5 & 0.6448 & 0.6257 & 2.97 & 1.2653 & 1.3017 & -2.88 \\
\hline & & 20 & 0.8394 & 0.8298 & 1.14 & 1.3535 & 1.3793 & -1.90 \\
\hline & & 100 & 0.8581 & 0.8565 & 0.19 & 1.4629 & 1.4645 & -0.11 \\
\hline & \multirow[t]{3}{*}{$50\left(10^{-2} w\right)$} & 5 & 0.4291 & 0.4401 & -2.56 & 0.9635 & 0.9309 & 3.38 \\
\hline & & 20 & 0.7053 & 0.7029 & 0.33 & 1.9994 & 2.0375 & -1.91 \\
\hline & & 100 & 1.1860 & 1.1919 & -0.50 & 2.1637 & 2.1372 & 1.22 \\
\hline
\end{tabular}

Table 15 Sixth casemaximum deflections (not considering nanotubes; $\left.V_{\underline{\mathrm{f}}}=0.6\right)$

\begin{tabular}{|c|c|c|c|c|c|}
\hline Stacking sequence & $a / h$ & $R / a$ & $17 \times 17$ grid & Point cloud & $\operatorname{Dev}(\%)$ \\
\hline \multirow[t]{9}{*}[0^{\circ}/90^{\circ}]{} & \multirow[t]{3}{*}{$10\left(10^{-4} w\right)$} & 5 & 2.9901 & 3.0262 & -1.21 \\
\hline & & 20 & 3.2386 & 3.2620 & -0.72 \\
\hline & & 100 & 3.2558 & 2.2566 & -0.03 \\
\hline & \multirow[t]{3}{*}{$20\left(10^{-3} w\right)$} & 5 & 1.8478 & 1.7819 & 3.57 \\
\hline & & 20 & 2.4134 & 2.4507 & -1.55 \\
\hline & & 100 & 2.4600 & 2.4837 & -0.96 \\
\hline & \multirow[t]{3}{*}{$50\left(10^{-2} w\right)$} & 5 & 1.2750 & 1.3021 & -2.13 \\
\hline & & 20 & 3.3915 & 3.4597 & -2.01 \\
\hline & & 100 & 3.8030 & 3.8107 & -0.20 \\
\hline \multirow[t]{9}{*}[0^{\circ}/90^{\circ}/0^{\circ}]{} & \multirow[t]{3}{*}{$10\left(10^{-4} w\right)$} & 5 & 2.1263 & 2.1463 & -2.09 \\
\hline & & 20 & 2.1024 & 2.3167 & -2.78 \\
\hline & & 100 & 2.2541 & 2.4456 & 0.15 \\
\hline & \multirow[t]{3}{*}{$20\left(10^{-3} w\right)$} & 5 & 1.6029 & 1.6251 & -1.38 \\
\hline & & 20 & 1.9345 & 1.9105 & 1.24 \\
\hline & & 100 & 2.3992 & 2.4000 & -0.03 \\
\hline & \multirow[t]{3}{*}{$50\left(10^{-2} w\right)$} & 5 & 1.1159 & 1.0914 & 2.20 \\
\hline & & 20 & 2.1237 & 2.0599 & 3.00 \\
\hline & & 100 & 2.3562 & 2.3087 & 2.02 \\
\hline
\end{tabular}

\section{Conclusions}

With the present work, the authors intended to establish a continuous integrated process of surfaces reconstruction to finite-element analysis. To this objective, one has implemented meshless methods based on different types of radial basis functions which were found to be the more adequate for each of this process stage.

Thus, in the first stage of this process, one proceeds to the reconstruction of shells' surfaces, described by 
Table 16 Seventh case-maximum deflections $\left(w^{\mathrm{CN}}=0.04 ; V_{\mathrm{f}}=0.6\right)$

\begin{tabular}{|c|c|c|c|c|c|c|c|c|}
\hline \multirow[t]{2}{*}{ Stacking sequence } & \multirow[t]{2}{*}{$a / h$} & \multirow[t]{2}{*}{$R / a$} & \multicolumn{3}{|c|}{ Single-walled CNT } & \multicolumn{3}{|c|}{ Multi-walled CNT } \\
\hline & & & $17 \times 17$ grid & Point cloud & $\operatorname{Dev}(\%)$ & $17 \times 17$ grid & Point cloud & $\operatorname{Dev}(\%)$ \\
\hline \multirow[t]{9}{*}[0^{\circ}/90^{\circ}]{} & \multirow[t]{3}{*}{$10\left(10^{-4} w\right)$} & 5 & 1.7181 & 1.6892 & 1.68 & 4.1859 & 4.1633 & 0.54 \\
\hline & & 20 & 1.8733 & 1.8486 & 1.32 & 4.5501 & 4.6297 & 2.91 \\
\hline & & 100 & 1.8831 & 1.8969 & -0.73 & 4.5814 & 4.5983 & -0.37 \\
\hline & \multirow[t]{3}{*}{$20\left(10^{-3} w\right)$} & 5 & 1.0615 & 1.0256 & 3.38 & 2.6134 & 2.5585 & 2.10 \\
\hline & & 20 & 1.4274 & 1.4288 & -0.10 & 3.3938 & 3.4551 & -1.81 \\
\hline & & 100 & 1.4491 & 1.4547 & -0.39 & 3.4850 & 3.5105 & -0.73 \\
\hline & \multirow[t]{3}{*}{$50\left(10^{-2} w\right)$} & 5 & 0.8626 & 0.8554 & 0.83 & 1.6939 & 1.6542 & 2.34 \\
\hline & & 20 & 1.9851 & 2.0234 & -1.93 & 4.7907 & 4.6899 & 2.10 \\
\hline & & 100 & 2.2620 & 2.2188 & 1.91 & 5.3586 & 5.3378 & 0.39 \\
\hline \multirow[t]{9}{*}[0^{\circ}/90^{\circ}/0^{\circ}]{} & \multirow[t]{3}{*}{$10\left(10^{-4} w\right)$} & 5 & 1.6114 & 1.5841 & 1.70 & 2.4750 & 2.4587 & 0.66 \\
\hline & & 20 & 1.5984 & 1.5750 & 1.46 & 2.7748 & 2.8321 & -2.07 \\
\hline & & 100 & 1.8374 & 1.8229 & 0.79 & 2.8551 & 2.8753 & -0.71 \\
\hline & \multirow[t]{3}{*}{$20\left(10^{-3} w\right)$} & 5 & 0.8517 & 0.8351 & 1.95 & 1.6416 & 1.6225 & 1.16 \\
\hline & & 20 & 1.2393 & 1.2561 & -1.36 & 2.2046 & 2.2536 & -2.22 \\
\hline & & 100 & 1.2417 & 1.2502 & -0.68 & 2.2301 & 2.2777 & -2.13 \\
\hline & \multirow[t]{3}{*}{$50\left(10^{-2} w\right)$} & 5 & 0.5433 & 0.5374 & 1.09 & 2.1640 & 2.1242 & 1.84 \\
\hline & & 20 & 0.5856 & 0.5990 & -2.29 & 3.1994 & 3.1220 & 2.42 \\
\hline & & 100 & 1.8202 & 1.8009 & 1.06 & 3.9598 & 4.0025 & -1.08 \\
\hline
\end{tabular}
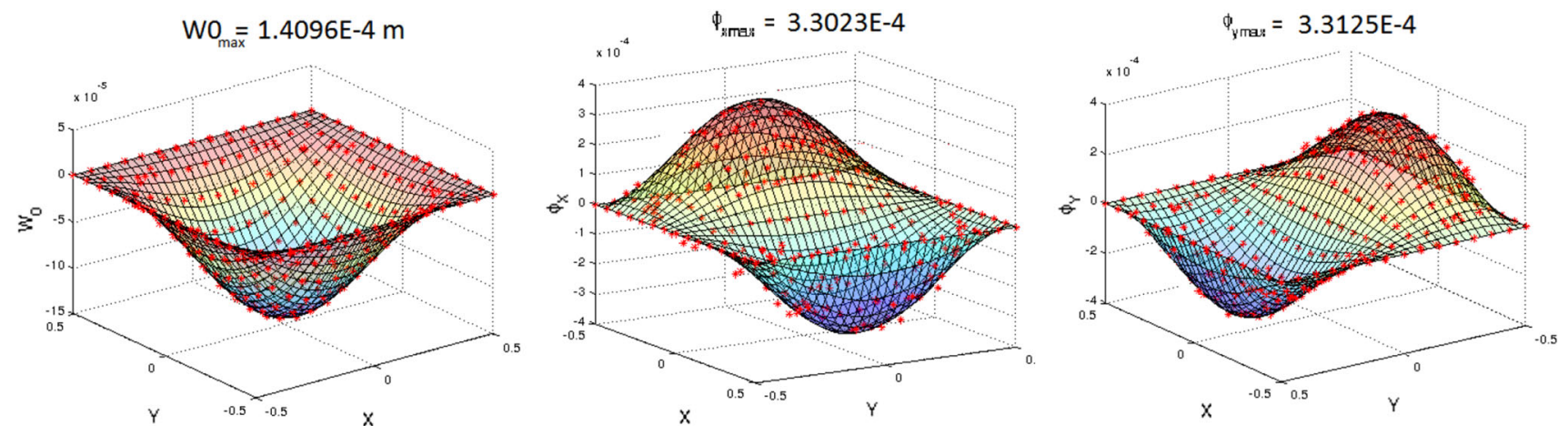

Fig. 9 Representations of mid-plane transverse displacement (left), the rotations around $x$-axis (centre) and $y$-axis (right)

unstructured 3D point-clouds which may be acquired via 3D laser scanning, and in the second phase, and for illustration purposes, a set of shell structures is analysed through Donnell first-order shear deformation theory.

The results obtained either considering the reconstruction phase or the static analysis phase have shown to be very promising. The inter-connection of the methods and the association of these two phases have also proven to provide an effective computational tool, allowing for a complete workflow from the reconstruction phase to the analysis one.

As far as the author's knowledge, there is not any published work proposing and illustrating the performance of a similar integrated methodology. This combined methodology can provide several advantages over others available through different independent applications, which always require an intermediate processing to turn compatible their output/input data.

It is worth mentioning that the present methodology is very pertinent in many science fields, in the sense that it allows to ease not just the optimization of databases which include the information about the real condition of an existing structure, but also the analysis of such structure.

Acknowledgements The authors wish to acknowledge the financial support given by FCT/MEC through Project PTDC/ATP-AQI/5355/ 2012 and Project LAETA-UID/EMS/50022/2013. 
Open Access This article is distributed under the terms of the Creative Commons Attribution 4.0 International License (http://crea tivecommons.org/licenses/by/4.0/), which permits unrestricted use, distribution, and reproduction in any medium, provided you give appropriate credit to the original author(s) and the source, provide a link to the Creative Commons license, and indicate if changes were made.

\section{References}

Alexa M, Behr J, Cohen-Or D, Fleishman S, Levin D, Silva CT (2001) Point set surfaces. In: IEEE visualization, pp 21-28

Barazzetti L, Banfi F, Brumana R, Gusmeroli G, Oreni D, Previtali M, Roncoroni F, Schiantarelli G (2015) BIM from laser clouds and finite element analysis: combining structural analysis and geometric complexity. In: The international archives of the photogrammetry, remote sensing and spatial information sciences, vol XL-5/W4, 2015 3D virtual reconstruction and visualization of complex architectures, Avila, pp 345-350

Beatson RK, Cherrie JB, Ragozin DL (2001) Fast evaluation of radial basis functions: methods for four-dimensional polyharmonic splines. SIAM J Sci Math Anal 32(6):1272-1310

Bentley J (1975) Multidimensional binary search trees used for associate searching. CACM 18(9):509-517

Bernardo GMS, Loja MAR (2015) Reconstruction of surfaces from unstructured points clouds, using compactly-supported radial basis functions. In: 2nd international conference on numerical and symbolic computation

Bernardo GMS, Loja MAR (2015) Static and free vibrations behavior of particulate composite plates using radial basis functions. In: 2nd international conference on numerical and symbolic computation

Bosch FN, Guillemet A, Turkan Y, Haas CT, Haas R (2014) Tracking the built status of MEP works: assessing the value of a Scan-vsBIM system. J Comput Civil Eng 28(4):1-28

Carr C, Fright WR, Beatson RK (1997) Surface interpolation with radial basis functions for medical imaging. IEEE Trans Med Imaging 16(1):96-107

Carr JC, Beatson RK, Cherrie JB, Mitchell TJ, Fright WR, McCallum BC, Evans TR (2001) Reconstruction and representation of 3D objects with radial basis functions. In: SIGGRAPH conference proceedings, pp 67-76

Castellazzi G, D’Altri AM, Bitelli G, Selvaggi I, Lambertini A (2015) From laser scanning to finite element analysis of complex buildings by using a semi-automatic procedure. Sensors 15:18360-18380

Chalmovianský P, Juttler B (2003) Filling holes in point clouds. In: Wilson XM, Martin R (eds) Math surfaces. Springer, Berlin, pp 196-212

Cheney E, Light W (1999) A course in approximation theory. Brooks Cole, Pacific Grove

Costa DMS, Loja MAR (2016) Assessing the static behavior of hybrid CNT-metal-ceramic composite plates. AIMS Mater Sci 3(3):808-831

Dinh HQ, Turk G, Slabaugh G (2002) Reconstructing surfaces by volumetric regularization using radial basis functions. IEEE Pattern Anal Mach Intell 24(10):1358-1371

Ferreira AJM, Roque CMC (2007) Jorge RMN. Natural frequencies of FSDT cross-ply composite shells by multiquadrics. Compos Struct 77:296-305

Ferreira AJM, Roque CMC (2009) New developments in the radial basis functions analysis of composite shells. Compos Struct 87(9):141-150

Ferreira AJM, Batra RC, Roque CMC, Qian LF, Martins PLS (2005) Static analysis of functionally graded plates using third-order shear deformation theory and a meshless method. Compos Struct 69(4):449-457

Hoppe H, DeRose T, Duchamp T, McDonald J, Stuetzle W (1992) Surface reconstruction from unorganized point clouds. In: SIGGRAPH proceedings, pp 71-78

$\mathrm{Hu}$ Y, Shenderova OA, Hu Z, Padgett CW, Brenner DW (2006) Carbon nanostructures for advanced composites. Rep Prog Phys 69:1847-1895

Huber D, Akinci B, Tang P, Adan A, Okorn B, Xuehan X (2010) Using laser scanners for modeling and analysis in architecture, engineering, and construction. Information and systems (CISS), pp 1-6

Kansa EJ (1990) Multiquadrics-a scattered data approximation scheme with applications to computational fluid dynamics, I: surface approximations and partial derivative estimates. Comput Math Appl 19(8/9):127-145

Krysl P, Belytshko T (2000) An efficient linear-precision partition of unity basis for unstructured meshless methods. Commun Numer Methods Eng 16:239-255

Morse BS, Yoo TS, Rheingans P, Chen DT, Subramanian (2001) Interpolating implicit surfaces from scattered surface data using compactly supported radial basis functions. In: IEEE international conference on shape modeling and applications, pp 89-98

Nealen A (2004) An as-short-as-possible introduction to the least squares, weighted least squares and moving least squares methods for scattered data approximation and interpolation. Technical Report, TU Darmstadt

Raffie M, He XQ (2014) Modeling and stress analysis of smart cnts/fiber/polymer multiscale composite plates. Int J Appl Mech 6(3): $1-23$

Reddy JN (2004) Mechanics of laminated composite plates and shells-theory and analysis, 2nd edn. CRC Press, Boca Raton

Rusinkiewicz S, Levoy M, Qsplat (2000) A multiresolution point rendering system for large meshes. In: Proceedings of SIGGRAPH 2000, pp 343-352

Savchenko VV, Pasko AA, Okunev OG, Kunii TL (1995) Function representation of solids reconstructed from scattered surface points and contours. Comput Graphics Forum 14(4):181-188

Szolomicki J (2015) Application of 3D laser scanning to computer model of historic buildings. In: Proceedings of the world congress on engineering and computer science, vol II, WCECS 2015, San Francisco

Taubin G (1991) Estimation of planar curves, surfaces, and nonplanar spaces curves defined by implicit equations with applications to edge and range image segmentation. IEEE Trans Pattern Anal Mach Intell 13:11

Taubin G (1993) An improved algorithm for algebraic curve and surface fitting. In: Proceedings fourth international conference on computer vision, pp 658-665

Thostenson ET, Li C, Chou T-W (2005) Review: nanocomposites in context. Compos Sci Technol 65:491-516

Tornabene F, Fantuzzi N, Viola E, Ferreira AJM (2013) Radial basis functions method applied to doubly-curved laminated composite shells and panels with a general higher-order equivalent single layer formulation. Composites Part B 55(13):642-659

Tornabene F, Fantuzzi N, Bacciocchi M, Viola E (2016) Effect of agglomeration on the natural frequencies of functionally graded carbon nanotube-reinforced laminated composite doubly curved shells. Compos B 89:187-218

Turk G, Obrien J (2002) Modelling with implicit surfaces that interpolate. ACM Trans Graphics 21:4

Wendland H (1995) Piecewise polynomial, positive definite and compactly supported radial basis functions of minimal degree. AICM 4:389-396 
Wittek A, Grosland NM, Joldes GM, Magnotta V, Miller K (2016) From finite element meshes to clouds of points: a review of methods for generation of computational biomechanics models for patient-specific applications. Ann Biomed Eng 44(1):3-15
Yang H, Xu X, Neumann I (2014) The benefit of 3d laser scanning technology in the generation and calibration of fem models for health assessment of concrete structures. Sensors 14:21889-21904 